\title{
MAGNETIC STRUCTURE OF RNiGe AND RPdSn COMPOUNDS*
}

\author{
G. ANDré, F. BOURÉE \\ Laboratoire Léon Brillouin (CEA-CNRS) \\ CEN-Saclay, 91191 Gif-sur-Yvette Cedex, France \\ A. Bombik, A. Oleś, W. Sikora \\ Department of Physics and Nuclear Technology, Academy of Mining and Metallurgy \\ Reymonta 17, 30-059 Kraków, Poland \\ M. Kolenda, A. Szytula \\ Institute of Physics, Jagiellonian University, Reymonta 4, 30-059 Kraków, Poland
}

\section{A. PACYNa}

Institute of Nuclear Physics, Radzikowskiego 152, 31-342 Kraków, Poland

\section{AND A. ZYGMUNT}

W. Trzebiatowski Institute for Low Temperatures and Structure Research Polish Academy of Sciences, Okólna 2, 50-950 Wrocław, Poland

Magnetic properties including magnetic structure of ternary RNiGe $(\mathrm{R}=\mathrm{Tb}-\mathrm{Er})$ and $\mathrm{RPdSn}(\mathrm{R}=\mathrm{Ce}, \mathrm{Tb}-\mathrm{Ho})$ compounds are determined. In low temperatures an antiferromagnetic ordering is observed. For compounds with $\mathrm{R}=\mathrm{Tb}-\mathrm{Er}$ the magnetic structure changes with temperature.

PACS numbers: $75.25 .+z$

\section{Introduction}

The equiatomic ternary rare earth intermetallic compounds with the general formula RTX ( $\mathrm{R}=$ rare earth, $\mathrm{T}=n d$ transition element and $\mathrm{X}=\mathrm{Si}, \mathrm{Ge}, \mathrm{Sn})$ are intensively investigated in recent years [1].

In this work we present results of neutron diffraction and magnetometric measurements undertaken in order to determine the magnetic structure of $\mathrm{RNiGe}$ $(\mathrm{R}=\mathrm{Tb}, \mathrm{Dy}, \mathrm{Ilo}$ and $\mathrm{Er})$ and $\mathrm{RPdSn}(\mathrm{R}=\mathrm{Ce}, \mathrm{Tb}, \mathrm{Dy}$, and $\mathrm{Ho})$.

*This work was partially supported by the grants 2-0083-91-01 and 2-2438-92-03 of the Committee for Scientific Research. 


\section{Experiment}

The RNiGe and RPdSn samples are synthetized by arc melting of stoichiometric amounts of high purity components. The samples are subsequently annealed in vacuum for $100 \mathrm{~h}$ at $800^{\circ} \mathrm{C}$. The X-ray diffraction measurements were carried out at room temperature and they show that all samples are in single phases. The magnetometric measurements were carried out using an RH Cahn balance and vibrating magnetometer in the temperature range of $2-300 \mathrm{~K}$.

Neutron diffraction data were obtained by means of the neutron powder diffractometer G4.1 installed at the Orphée reactor (Laboratoire Léon Brillouin, Saclay) with an incident neutron wavelength of $2.4268 \AA$.

\section{Results}

The reflections observed in neutron diffraction patterns obtained at $300 \mathrm{~K}$ for both series are characteristic for the TiNiSi structure.

The temperature dependence of the magnetic susceptibility of all samples exhibits maxima which are characteristic of a transition to an antiferromagnetic state. The determined values of the Néel temperature are listed in Table. In TbNiGe, TbPdSn and DyPdSn below $T_{N}$ the additional phase transition is observed (see Fig. 1). Above $T_{N}$ the reciprocal magnetic susceptibility obeys the Curie-Weiss law. The values of paramagnetic Curie temperature and the effective magnetic moments $\mu_{\text {eff }}$ are given in Table.

TABLE

Magnetic data for RNiGe and RPdSn compounds.

\begin{tabular}{c|c|c|c|c|c|c|c}
\hline \hline Compound & $T_{\mathrm{N}}[\mathrm{K}]$ & $T_{\mathrm{t}}[\mathrm{K}]$ & $\Theta_{\mathrm{p}}[\mathrm{K}]$ & \multicolumn{2}{|c|}{$\mu_{\mathrm{eff}}\left[\mu_{\mathrm{B}} / \mathrm{R}^{3+}\right]$} & \multicolumn{2}{|c}{$\mu\left[\mu_{\mathrm{B}} / \mathrm{R}^{3+}\right]$} \\
\cline { 4 - 7 } & & & & exp. & theor. & exp. & theor. \\
\hline TbNiGe & 18.5 & 10 & +4.1 & 8.97 & 9.72 & 9.1 & 9.0 \\
DyNiGe & 4.7 & 3.5 & -8.2 & 10.42 & 10.62 & 7.54 & 10.0 \\
HoNiGe & 2.75 & 2.2 & -2.6 & 11.2 & 10.61 & 9.98 & 10.0 \\
ErNiGe & 2.9 & 2.6 & -1.4 & 9.19 & 9.58 & 9.05 & 9.0 \\
CePdSn & 7.0 & & -6.3 & 2.7 & 2.54 & 0.88 & 2.14 \\
TbPdSn & 19 & 10 & -11 & 10.1 & 9.72 & 7.6 & 9.0 \\
DyPdSn & 10 & -7 & -7 & 10.5 & 10.65 & 7.6 & 10.0 \\
HoPdSn & 3.7 & & -7.5 & 10.7 & 10.61 & 9.0 & 10.0
\end{tabular}

In low temperatures in the neutron diffraction patterns a large number of additional peaks of magnetic origin is observed.

The analysis of angular positions and intensities of magnetic peaks in the neutron diffraction-patterns indicates the following model of the magnetic structure: 


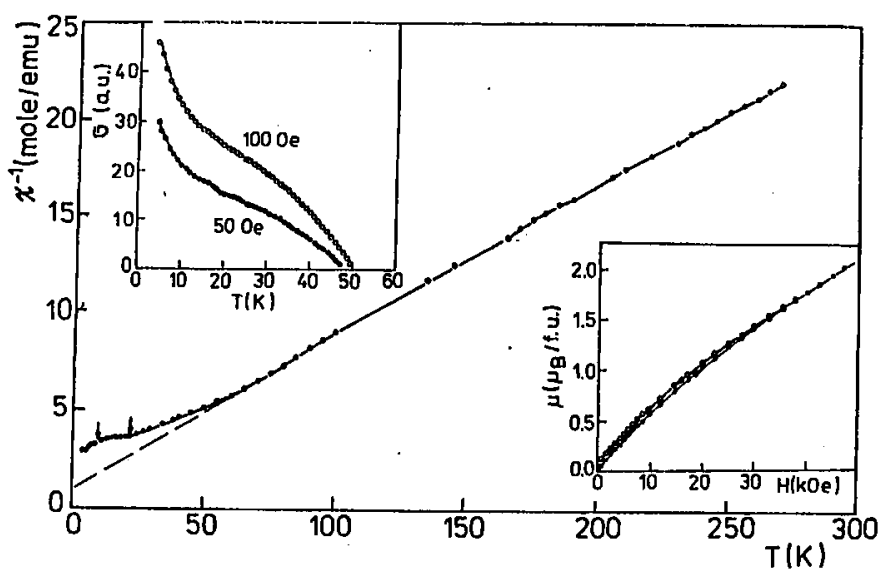

Fig. 1. The temperature dependence of the reciprocal magnetic susceptibility of TbPdSn. The arrows indicate $T_{\mathrm{N}}$ and $T_{\mathrm{t}}$. The insets present the low temperature part of temperature dependence of magnetization (left) and the magnetization curve at $T=4.2 \mathrm{~K}$ in the magnetic field up to $50 \mathrm{kOe}$ (right).
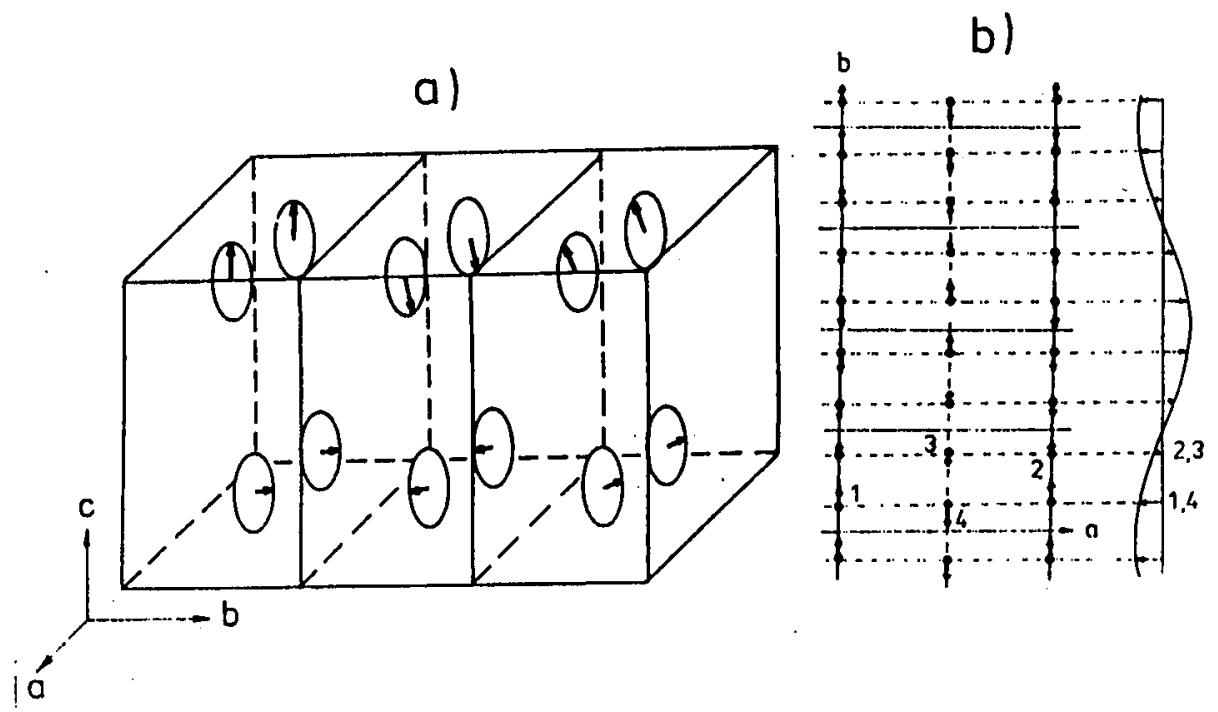

Fig. 2. Magnetic structure of CePdSn (a) and DyPdSn (b).

- In case of RNiGe compounds the TbNiGe and DyNiGe show a square modulated structure with the wave vector $k=(2 / 3,1 / 3,0)$ at low temperature. As the temperature rises a change into a sinusoidally modulated structure in TbNiGe and into a cycloidal spiral structure in DyNiGe is observed. In both structures and in both phases the magnetic moments are parallel to the $c$-axis. At low temperatures the magnetic structures with the wave vector $k_{1}=(1 / 2,0,1 / 2)$ in 
HoNiGe and $k_{2}=(0,1 / 2,0)$ in ErNiGe were discovered. An increase in the temperature caused a change to a modulated magnetic ordering with the wave vector $k_{1}=(0.48,0.23,0.42)$ in HoNiGe and $k_{2}=(0,0.5,0.0837)$ in ErNiGe. The magnetic moment of Ho atoms is parallel to the $a$-axis whereas in ErNiGe the magnetic moment of $\mathrm{Er}^{3+}$ ions forms an angle with the $a$-axis at low temperature.

- In case of RPdSn compounds the CePdSn has a simple spiral magnetic structure with the wave vector $k=(0,0.473,0)$ in the temperature range $T=1.4 \mathrm{~K}$ to $T_{\mathrm{N}}=7.3 \mathrm{~K}$ (see Fig. $2 \mathrm{a}$ ).

The Tb moments in TbPdSn order in a sine-wave-modulated magnetic spin arrangement with the wave vector $k=(0,0.25,0)$ in the temperature range $T_{\mathrm{t}}=$ $10 \mathrm{~K}$ and $T_{\mathrm{N}}=19 \mathrm{~K}$ and with the $k=(0,0.25,0.075)$ below $T_{\mathrm{t}}$.

In DyPdSn compound $\mathrm{Dy}^{3+}$ ions order antiferromagnetically below $T_{\mathrm{N}}=$ $10.5 \mathrm{~K}$ in a sine modulated spin arrangement (see Fig. 2b) of the magnetic moments with the wave vector $k=(0,0.25,0)$. Below $T_{\mathrm{t}}=7 \mathrm{~K}$ two magnetic structures coexist: the sine modulated observed below $T_{\mathrm{t}}$ and a spiral structure. In both structures magnetic moments are parallel to the $b$-axis. The magnetic moment located in the crystal unit cell are coupled in a $S_{1}+S_{2}-S_{3}-S_{4}$ order.

In HoPdSn compound at low temperature a coexistence of two magnetic structures is observed. One with the wave vector $k_{1}=(0,0.268,0)$ and the second one with $k_{2}=(0.333,0.0635,0.0748)$.

\section{Discussion}

All compounds discussed in this work crystallize in the orthorhombic TiNiSi-type crystal structure and are antiferromagnets in low temperatures. The magnetic moments in these compounds are almost exclusively due to localized $4 f$-electrons. The magnetic ordering in rare-earth intermetallic compounds may be explained as a competition of integral interactions of the RKKY-type and a magneto-crystalline anisotropy caused by an influence of the crystalline electric field on $4 f$-electrons. The RKKY-type exchange interactions favour a long range oscillatory antiferromagnetic ordering while the magneto-crystalline anisotropy favours a uniaxial magnetic ordering.

\section{References}

[1] A. Szytuła, in: Handbook of Magnetic Materials, Ed. K.H.J. Buschow, Vol. 6, North-Holland, Amsterdam 1991, p. 83. 